\title{
Cone Beam Computed Tomography (CBCT) is Superior to Orthopantomogram (OPG) in Interpreting the Imaging Characteristics of Medication-Related Osteonecrosis of the Jaw (MRONJ and More Stage-Sensitive: A Prospective Assessment in 98 Cancer Patients
}

OPEN ACCESS

${ }^{*}$ Correspondence:

Dimitra Galiti, Oral Diagnosis and

Radiology Clinic, Dental School,

National and Kapodistrian University of Athens, Anastaseos 23, Holargos 15561, Athens, Greece, Tel: 306938039553;

E-mail:dimigalitis@hotmail.com Received Date: 05 Aug 2021 Accepted Date: 31 Aug 2021 Published Date: 10 Sep 2021

Citation:

Galiti D, Karayianni A, Psyrri A, Tsiklakis K. Cone Beam Computed

Tomography (CBCT) is Superior

to Orthopantomogram (OPG) in Interpreting the Imaging Characteristics of Medication-Related Osteonecrosis of the Jaw (MRONJ and More StageSensitive: A Prospective Assessment in 98 Cancer Patients. World J Surg

Surgical Res. 2021; 4: 1335.

Copyright $\odot 2021$ Dimitra Galiti. This is an open access article distributed under

the Creative Commons Attribution

License, which permits unrestricted use, distribution, and reproduction in any medium, provided the original work is properly cited.

\begin{abstract}
Dimitra Galiti ${ }^{1 *}$, Aikaterini Karayianni ${ }^{1}$, Amanda Psyrri ${ }^{2}$ and Kostas Tsiklakis ${ }^{1}$
${ }^{1}$ Oral Diagnosis and Radiology Clinic, Dental School, National and Kapodistrian University of Athens, Greece

${ }^{2}$ Department of Oncology, B Propaideytiki Pathology Clinic, Peripheral General Hospital of Athens Attikon, School of Medicine, National and Kapodistrian University of Athens, Greece
\end{abstract}

\begin{abstract}
Objectives: To prospectively evaluate the imaging characteristics of Medication-Related Osteonecrosis of the Jaw (MRONJ), observed in the Orthopantomogram (OPG) and in Cone Beam Computed Tomography (СBCT), in patients with cancer.
\end{abstract}

Materials and Methods: Cancer patients, who received Bone Targeting Agents (BTAs) and developed MRONJ, were prospectively included in the study. MRONJ was staged following the American Association of Oral and Maxillofacial Surgery (AAOMS) 2014 criteria. Four predefined radiological findings, osteolytic changes, sclerosis, periosteal reaction, and sequestration were assessed and were scored as "absent", "localized", "extensive" and "beyond the alveolar bone".

Results: Ninety-eight cancer patients were enrolled. Eighty patients (81.6\%) received BTAs for over a year; 19 (19.4\%) received BTAs between 13 to 24 months and 61 patients $(62.2 \%)$ received BTAs for over 24 months. The majority of the patients $(n=58,59.2 \%)$ had stage $2 \mathrm{MRONJ}$ and $35(35.7 \%)$ were at MRONJ stage 3.

Fifty-two (53.06\%) patients were assessed with OPG and 89 (90.81\%) with CBCT and CBCT was found more effective in detecting sclerosis and sequestration than OPG.

Forty-three $(43.87 \%)$ patients were assessed with both OPG and CBCT and were available for comparisons between OPG and CBCT. CBCT was significantly more accurate than OPG in interpreting the "beyond the alveolar bone" osteolytic changes, sclerosis, periosteal reaction and sequestration, in all MRONJ stages. P-value was $0.0002,<0.0001,0.0027$ and 0.0009 respectively.

Furthermore, $\mathrm{CBCT}$ was significantly more accurate than OPG in interpreting the "extensive" and "beyond the alveolar bone" imaging characteristics, when comparisons included the patients with early stages of MRONJ, 1 and 2 and the patients with stage 3 of MRONJ.

Conclusion: $\mathrm{CBCT}$ is superior and more stage-sensitive to OPG in interpreting and staging MRONJ and can be particularly useful to achieve an early diagnosis of MRONJ.

Keywords: Bone targeting agents; Cone-Beam computer tomography; Medication-Related osteonecrosis of the jaw; Orthopantomogram

\section{Introduction}

Medication-Related Osteonecrosis of the Jaw (MRONJ) is defined as a complication, which affects the jawbone of patients that meet all of the following criteria: (a) patients were in the past or are now on treatment with bone targeting agents and/or antiangiogenics, (b) they have exposed bone or bone that can be probed through an intraoral or extraoral fistula in the maxillofacial region for more than 8 weeks, and (c) they have no history of radiation therapy or obvious metastatic disease to the jaws [1-5]. More than $90 \%$ of the MRONJ cases occur in cancer patients, who receive high doses of BTAs, with an incidence between $1.1 \%$ to $1.3 \%$ for the first year of administration 


\section{$[2-4]$.}

Imaging characteristics of the jaws support the clinical diagnosis and are recommended to determine the stage and to effectively manage the MRONJ (Table 1) [1-5]. The International Task Force on MRONJ suggests that intraoral and panoramic radiographs are useful in evaluating the presence of changes of MRONJ, such as thickening of lamina dura, increased trabecular density of the alveolar bone or widening of the periodontal ligament space [2]. Moreover, they suggest that Computed Tomography (CT) offers the advantages of assessing maxilla and mandible trabecular architecture, cortical integrity, periosteal bone and early fistula formation. Cone-Beam CT (CBCT) is recommended as being particularly useful displaying with higher resolution the jawbone. CBCT is advantageous over OPG, as panoramic radiographs produce a two-dimension image and do not allow for a full evaluation of the extent of the lesion. The Japanese Allied Committee on Osteonecrosis of the Jaw suggests in patients that are clinically suspected of having MRONJ, to perform CBCT for detecting early changes in trabecular and cortical bones and in assessing sequestra, fistulas and periosteal responses [4].

However, only a few articles have been published regarding the imaging characteristics of MRONJ and are mostly based on findings from small and retrospective studies with a mixed population of osteoporosis and cancer patients and with MRONJ staging based only on clinical criteria [6-11]. Osteolytic lesions, osteosclerosis and bone sequestrum were reported in both OPG and CBCT in two studies, while sclerosis, lytic changes, periosteal reactions and sequestration were more frequent in clinical stage 3 of MRONJ in other studies [611]. One study concluded that MRONJ has characteristic CT image findings, such as bone sequestrum, bone perforation and periosteal reaction that could be useful for its assessment [7]. Walton et al. reported that $\mathrm{CT}$ scans were useful to accurately define the extent of osteolytic lesions and the stage [11]. Furthermore, the imaging characteristics associated with increased risk for the development of MRONJ were evaluated in four studies. All 4 studies included mixed patient populations and two were retrospective, one was pilot, and only one was prospective study. Osteosclerosis, periodontal ligament widening, periodontal disease and bone sequestrum were related with increased risk for MRONJ [12-15].

A critical review reported the scarcity of quantitative studies to assess the role of imaging techniques in MRONJ [16]. Furthermore, in a recent meta-analysis, which involved 29 studies with 1,133 patients, who received antiresorptive therapy, and were examined with intraoral and/or OPG radiographs or CBCT, the prevalence of radiographic findings was high, however, the level of evidence of the studies was very low [17].

It is apparent that the radiological findings for assessing and accurate staging of MRONJ are inadequately described. In that respect, we aimed to prospectively evaluate the imaging characteristics of Orthopantomogram (OPG) and Cone Beam Computed Tomography (CBCT) and compare them by stages of Medication-Related Osteonecrosis of the Jaw (MRONJ) in patients with cancer.

\section{Materials and Methods}

A prospective, 30-month follow-up, observational study was conducted between June 2017 and October 2019, aiming to include a convenient sample of 100 patients. Eligible participants for this analysis were cancer patients diagnosed with MRONJ. Patients with MRONJ and with an insufficient, non-evaluable, OPG or CBCT were excluded.

All study participants received information about the study, agreed to participate and signed the informed consent. The study was approved by the Committee of Research and Ethics of the Dental School, National and Kapodistrian University of Athens, reference number 391.

The demographic and underlying disease characteristics were recorded. MRONJ was defined according to the American Association of Oral and Maxillofacial Surgeons (AAOMS) updated criteria of 2014, as shown in Table 1 [1]. Staging of MRONJ was based on the combination of clinical data and imaging findings of OPG and/or CBCT. Administrative reasons did not allow for all patients' assessment with both OPG and CBCT. For each OPG and CBCT, the imaging characteristics were first assessed by the Radiology Department of the Dental School or by patients' private radiologist (a written report was given) and then by the first author.

The following four radiographic findings were evaluated: osteolytic lesions, sclerosis (dense bone), periosteal reaction (subperiosteal bone deposition), and sequestration (bone sequestrum). The findings were scored as "absent" (0), as "localized" (1), when changes were confined within the alveolar bone and within one tooth dimension mesiodistally from the site of bone exposure, as "extensive" (2), when changes were confined within the alveolar bone and involved the bone of two teeth or multiple sites, and as "in and beyond the alveolar bone" (3), when changes were observed extending "beyond the alveolar bone" and included the involvement of the sinus floor, nasal walls, inferior alveolar canal, inferior mandibular border, and mandibular fracture.

\section{Statistical analysis}

Initial analysis was based on descriptive statistics. Absolute and relative frequencies were provided for categorical variables, while continuous were described by median, minimum and maximum. The difference in distribution of OPG and CBCT findings in patients who had both types of examination was assessed using the Wilcoxon Signed Rank Test. All tests were 2-sided, and the level of significance was set to $\alpha=0.05$. The entire statistical analysis was conducted with Stata 15.1 IC [18].

\section{Results}

\section{Patients and disease characteristics}

Ninety-eight patients with MRONJ were enrolled. Table 2 shows the patients' demographics and underlying disease characteristics at enrolment. Breast cancer was most common (48\%), followed by other malignancies. Nineteen patients received a BTA between 12 to 24 months and 61 for over 2 years. At enrolment, all patients had interrupted their BTA therapy.

Table 3 shows the MRONJ location, stage and risk factors in our study population. Thirty-two (32.7\%) patients had MRONJ in the maxilla, $52(53.1 \%)$ in the mandible and $14(14.3 \%)$ in both the maxilla and the mandible. Five patients $(5.1 \%)$ were classified as stage 1, 58 (59.2\%) had MRONJ stage 2 and 35 (35.7\%) were at MRONJ stage 3. Both clinical and imaging characteristics were used for the staging. Furthermore, tooth extraction was most often (48\%) reported as a preceding event for MRONJ, followed by periodontal disease, and other risk factors. Tooth pain was reported as the reason for dental extraction in $28(28.6 \%)$ patients. 
Table 1: Radiographic findings by stage of MRONJ as per diagnostic guidelines [AAOMS 2014].

\begin{tabular}{|c|c|c|c|}
\hline \multicolumn{4}{|c|}{ MRONJ STAGE } \\
\hline Stage 0 & Stage 1 & Stage 2 & Stage 3 \\
\hline \multicolumn{4}{|c|}{ Clinical definitions } \\
\hline $\begin{array}{l}\text {-Without clinical evidence of } \\
\text { necrotic bone } \\
\text {-With nonspecific clinical findings } \\
\text {-With symptoms }\end{array}$ & $\begin{array}{l}\text {-Exposed and necrotic bone } \\
\text { or fistula probing the bone } \\
\text {-Asymptomatic patients } \\
\text {-No evidence of infection }\end{array}$ & $\begin{array}{l}\text {-Exposed and necrotic bone or fistula probing the bone } \\
\text {-Infection evident by pain and erythema at the site of } \\
\text { the exposed bone with or without purulent drainage }\end{array}$ & $\begin{array}{l}\text {-Exposed and necrotic bone or fistula } \\
\text { probing the bone } \\
\text {-Symptoms of pain and infection } \\
\text {-Extraoral fistula } \\
\text {-Oral antral or oral nasal } \\
\text { communication }\end{array}$ \\
\hline \multicolumn{4}{|c|}{ Radiographic features } \\
\hline $\begin{array}{l}\text {-Alveolar bone loss, resorption not } \\
\text { attributed to periodontal disease } \\
\text {-Trabecular pattern changes } \\
\text {-Dense bone } \\
\text {-Lamina dura thickening } \\
\text {-Decreased periodontal ligament } \\
\text { space }\end{array}$ & $\begin{array}{l}\text {-Radiological findings } \\
\text { localized to the alveolar bone }\end{array}$ & -Radiological findings localized to the alveolar bone & $\begin{array}{l}\text {-Findings extending beyond the region } \\
\text { of alveolar bone } \\
\text {-Pathologic fracture } \\
\text {-Osteolysis extending to the inferior } \\
\text { border of the mandible or sinus floor }\end{array}$ \\
\hline
\end{tabular}

Table 2: Patients' demographics and underlying disease characteristics at enrolment.

\begin{tabular}{|c|c|}
\hline & Cancer patients \\
\hline & $N=98$ \\
\hline Age, median (range) & $71(47-89)$ \\
\hline Female, n (\%) & $55(56.1)$ \\
\hline Cancer, n (\%) & $98(100.0)$ \\
\hline Breast Ca & $47(48.0)$ \\
\hline Prostate $\mathrm{Ca}$ & $25(25.5)$ \\
\hline Multiple Myeloma & $16(16.3)$ \\
\hline Kidney Ca & $6(6.1)$ \\
\hline \multirow{2}{*}{$\begin{array}{l}\text { Lung Ca } \\
\text { Colon Ca }\end{array}$} & $3(3.1)$ \\
\hline & $1(1.0)$ \\
\hline \multicolumn{2}{|l|}{ Cancer therapy, n (\%) } \\
\hline Cytotoxic chemotherapy & $35(35.7)$ \\
\hline Targeted therapy & $42(42.9)$ \\
\hline Combined therapies & $13(13.3)$ \\
\hline Hormonal therapy & $46(46.9)$ \\
\hline \multicolumn{2}{|c|}{ Bone targeting agents (BTA), $n(\%)$} \\
\hline Zoledronic acid & $66(67.3)$ \\
\hline Denosumab - Xgeva & $32(32.6)$ \\
\hline \multicolumn{2}{|c|}{ Total duration of BTA, $n(\%)$} \\
\hline $1-12$ months & $18(18.4)$ \\
\hline 13-24 months & $19(19.4)$ \\
\hline$\geq 25$ months & $61(62.2)$ \\
\hline
\end{tabular}

Ca: Cancer

\section{Imaging characteristics}

Fifty-two (53.0\%) patients were assessed with OPG and 89 (90.8\%) with CBCT. Table 4 shows the imaging characteristics that were evaluated in OPG and CBCT. CBCT was more effective in detecting sclerosis and sequestration than OPG.

Table 5 shows the difference in distribution of OPG and CBCT findings in 43 (43.9\%) patients who had both types of examination. Out of the 16 "beyond the alveolar bone" osteolytic changes, observed with CBCT, only 9 were identified with OPG. The difference was significant $(\mathrm{P}=0.0002)$. Similarly, "beyond the alveolar bone" sclerosis, periosteal reaction, and sequestration were more frequently interpreted with СВСТ, with P-values being $<0.0001,0.0027$ and
Table 3: Location and stage of Medication Related Osteonecrosis of the Jaw (MRONJ) at assessment.

\begin{tabular}{|l|c|}
\hline & Cancer patients \\
\hline Location of MRONJ, $\mathbf{n}$ (\%) & \\
\hline Maxilla & $32(32.7)$ \\
\hline Mandible & $52(53.1)$ \\
\hline Maxilla and Mandible & $14(14.3)$ \\
\hline MRONJ stage, $\mathbf{n}$ (\%) & $5(5.1)$ \\
\hline 1 & $58(59.2)$ \\
\hline 2 & $35(35.7)$ \\
\hline 3 & $28(28.6)$ \\
\hline Risk factor for MRONJ, $\mathbf{( \% )}$ & $7(7.1)$ \\
\hline Tooth extraction & $18(18.4)$ \\
\hline Periodontal disease & $9(9.2)$ \\
\hline Denture use & $3(3.1)$ \\
\hline Implant & $1(1.0)$ \\
\hline Root fracture & $20(20.4)$ \\
\hline Missing & \\
\hline Reason for dental extraction, $\mathbf{n}(\%)$ & \\
\hline Tooth pain & \\
\hline To wear a denture or bridge & \\
\hline
\end{tabular}

0.0009 , respectively.

\section{OPG and CBCT findings by MRONJ stage}

Table 6 shows the difference in distribution of OPG and CBCT imaging characteristics among the 27 of the 43 patients, who had both types of examination, and were diagnosed with MRONJ stage 1 or 2. Out of the 16 "extensive" osteolytic changes that were found with $\mathrm{CBCT}$, only 10 were identified with OPG, $\mathrm{P}=0.0082$. Similarly, CBCT was superior to OPG in interpreting "extensive" sclerosis, periosteal reaction and sequestration, with $\mathrm{P}$-values being $<0.0001,0.0145$ and 0.0255 , respectively.

Table 7 shows the difference in distribution of OPG and CBCT imaging characteristics among the 16 of the 43 patients, who had both types of examination, and were diagnosed with MRONJ stage 3 . Out of the 16 "beyond the alveolar bone" osteolytic changes that were observed with CBCT, only 9 were identified with OPG. The difference 
Table 4: Imaging characteristics evaluated in OPG and CBCT.

\begin{tabular}{|c|c|c|c|c|}
\hline \multirow[t]{2}{*}{ Imaging characteristics } & \multicolumn{2}{|c|}{ OPG N=5252 } & \multicolumn{2}{|c|}{ СВСТ N=89 } \\
\hline & $\mathrm{N}$ & $\%$ & $\mathrm{~N}$ & $\%$ \\
\hline \multicolumn{5}{|l|}{ A. Osteolytic Lesion } \\
\hline 0. Absent & 1 & 1.92 & 0 & 0 \\
\hline 1. Localized & 23 & 44.23 & 18 & 20.22 \\
\hline 2. Extensive & 19 & 36.54 & 36 & 40.45 \\
\hline 3. Beyond the alveolar bone & 9 & 17.31 & 35 & 39.33 \\
\hline \multicolumn{5}{|l|}{ B. Sclerosis (Dense bone) } \\
\hline 0. Absent & 16 & 30.77 & 5 & 5.62 \\
\hline 1. Localized & 8 & 15.38 & 10 & 11.24 \\
\hline 2. Extensive & 22 & 42.31 & 30 & 33.71 \\
\hline 3. Beyond the alveolar bone & 6 & 11.54 & 44 & 49.44 \\
\hline \multicolumn{5}{|l|}{ C. Periosteal reaction } \\
\hline 0. Absent & 50 & 96.15 & 65 & 73.03 \\
\hline 1. Localized & 0 & 0 & 4 & 4.49 \\
\hline 2. Extensive & 1 & 1.92 & 9 & 10.11 \\
\hline 3. Beyond the alveolar bone & 1 & 1.92 & 11 & 12.36 \\
\hline \multicolumn{5}{|l|}{ D. Sequestration } \\
\hline 0. Absent & 48 & 92.31 & 53 & 59.55 \\
\hline 1. Localized & 0 & 0 & 12 & 13.48 \\
\hline 2. Extensive & 2 & 3.85 & 11 & 12.36 \\
\hline 3. Beyond the alveolar bone & 2 & 3.85 & 13 & 14.61 \\
\hline
\end{tabular}

Table 5: Differences in distribution of OPG and CBCT findings in 43 patients who had both types of examination.

\begin{tabular}{|c|c|c|c|c|c|}
\hline \multirow[t]{2}{*}{ Radiographic Features } & \multicolumn{2}{|c|}{ OPG } & \multicolumn{2}{|c|}{ СВСТ } & \multirow[t]{2}{*}{ P value* } \\
\hline & $\mathrm{N}$ & $\%$ & $\mathrm{~N}$ & $\%$ & \\
\hline A. Osteolytic Lesion & & & & & 0.0002 \\
\hline 0. Absent & 1 & 2.33 & 0 & 0 & \\
\hline 1. Localized & 17 & 39.53 & 11 & 25.28 & \\
\hline 2. Extensive & 16 & 37.21 & 16 & 37.21 & \\
\hline 3. Beyond the alveolar bone & 9 & 20.93 & 16 & 37.21 & \\
\hline B. Sclerosis (Dense bone) & & & & & $<0.0001$ \\
\hline 0. Absent & 12 & 27.91 & 1 & 2.33 & \\
\hline 1. Localized & 5 & 11.63 & 8 & 18.6 & \\
\hline 2. Extensive & 20 & 46.51 & 17 & 39.53 & \\
\hline 3. Beyond the alveolar bone & 6 & 13.95 & 17 & 39.53 & \\
\hline C. Periosteal reaction & & & & & 0.0027 \\
\hline 0. Absent & 41 & 95.35 & 33 & 76.74 & \\
\hline 1. Localized & 0 & 0 & 1 & 2.33 & \\
\hline 2. Extensive & 1 & 2.33 & 5 & 11.63 & \\
\hline 3. Beyond the alveolar bone & 1 & 2.33 & 4 & 9.3 & \\
\hline D. Sequestration & & & & & 0.0009 \\
\hline 0. Absent & 39 & 90.7 & 29 & 67.44 & \\
\hline 1. Localized & 0 & 0 & 7 & 16.28 & \\
\hline 2. Extensive & 2 & 4.65 & 2 & 4.65 & \\
\hline 3. Beyond the alveolar bone & 2 & 4.65 & 5 & 11.63 & \\
\hline
\end{tabular}

Table 6: Differences in distribution of OPG and CBCT findings in 27 patients who had stage 1 or 2

\begin{tabular}{|l|c|c|c|c|c|}
\hline \multicolumn{1}{|c|}{ Radiographic Features } & \multicolumn{2}{|c|}{ OPG } & \multicolumn{2}{c|}{ CBCT } & P value* \\
\hline & N & $\%$ & N & $\%$ & \\
\hline A. Osteolytic Lesion & & & & & 0.0082 \\
\hline 0. Absent & 1 & 3.7 & 0 & 0 & \\
\hline 1. Localized & 16 & 59.26 & 11 & 40.74 & \\
\hline 2. Extensive & 10 & 37.04 & 16 & 59.26 & \\
\hline 3. Beyond the alveolar bone & 0 & 0 & 0 & 0 & \\
\hline B. Sclerosis (Dense bone) & & & & & $<0.0001$ \\
\hline 0. Absent & 11 & 40.74 & 1 & 3.7 & \\
\hline 1. Localized & 4 & 14.81 & 8 & 29.63 & \\
\hline 2. Extensive & 11 & 40.74 & 10 & 37.04 & \\
\hline 3. Beyond the alveolar bone & 1 & 3.7 & 8 & 29.63 & \\
\hline C. Periosteal reaction & & & & & 0.0145 \\
\hline 0. Absent & 26 & 96.3 & 21 & 77.78 & \\
\hline 1. Localized & 0 & 0 & 1 & 3.7 & \\
\hline 2. Extensive & 1 & 3.7 & 3 & 11.11 & \\
\hline 3. Beyond the alveolar bone & 0 & 0 & 2 & 7.41 & \\
\hline D. Sequestration & & & & & 0.0255 \\
\hline 0. Absent & 0 & 0 & 0 & 0 & \\
\hline 1. Localized & & 0 & 4 & 14.81 & \\
\hline 2. Extensive & & & & \\
\hline 3. Beyond the alveolar bone & & & & \\
\hline Wilcoxon Signed-Rank Test & & & & \\
\hline
\end{tabular}

Table 7: Differences in distribution of OPG and CBCT findings in 16 patients who had stage 3.

\begin{tabular}{|l|c|c|c|c|c|}
\hline \multicolumn{1}{|c|}{ Radiographic Features } & \multicolumn{2}{|c|}{ OPG } & \multicolumn{2}{c|}{ CBCT } & P value* \\
\hline & N & $\%$ & N & $\%$ & \\
\hline A. Osteolytic Lesion & & & & & 0.0084 \\
\hline 0. Absent & 0 & 0 & 0 & 0 & \\
\hline 1. Localized & 1 & 6.25 & 0 & 0 & \\
\hline 2. Extensive & 6 & 37.5 & 0 & 0 & \\
\hline 3. Beyond the alveolar bone & 9 & 56.25 & 16 & 100 & \\
\hline B. Sclerosis (Dense bone) & & & & & 0.0146 \\
\hline 0. Absent & 1 & 6.25 & 0 & 0 & \\
\hline 1. Localized & 1 & 6.25 & 0 & 0 & \\
\hline 2. Extensive & 9 & 56.25 & 7 & 43.75 & \\
\hline 3. Beyond the alveolar bone & 5 & 31.25 & 9 & 56.25 & \\
\hline C. Periosteal reaction & & & & & 0.0836 \\
\hline 0. Absent & 15 & 93.75 & 12 & 75 & \\
\hline 1. Localized & 13 & 81.25 & 8 & 50 & \\
\hline 2. Extensive & 0 & 0 & 3 & 18.75 & \\
\hline 3. Beyond the alveolar bone & 1 & 6.25 & 2 & 12.5 & \\
\hline D. Sequestration & & & 0 & 0 & \\
\hline 0. Absent & & & & & \\
\hline 1. Localized & & & & & \\
\hline 2. Extensive & & & & & \\
\hline 3. Beyond the alveolar bone & & & & \\
\hline Wilcoxon Signed-Rank Test & & & & \\
\hline
\end{tabular}


was significant, $\mathrm{P}=0.0084$. Similarly, $\mathrm{CBCT}$ was superior to $\mathrm{OPG}$ in interpreting "beyond the alveolar bone" sclerosis and sequestration, with P-values being 0.0146 and 0.0148 , respectively. The difference was not significant in periosteal reaction, $\mathrm{P}=0.0836$.

\section{Discussion}

We prospectively assessed the imaging characteristics in 98 cancer patients with MRONJ. All study participants were diagnosed with stage 1, 2 and 3 according to the AAOMS 2014 guidelines. The diagnosis was based on both clinical and imaging characteristics [1]. No stage 0 was observed. MRONJ stage 0 was neither reported in the multicenter case registry study on MRONJ [19]. The AAOMS 2014 description of stage 0 does not fulfill the definition of MRONJ of exposed bone, and this is the reason for its lack of inclusion in clinical trials and studies [20]. MASCC/ISOO/ASCO expert panel shared those concerns and suggested considering stage 0 as an indicator for increased MRONJ risk [5].

In our study, the majority of our patients received BTAs for over a year; MRONJ was most frequently observed in the mandible and was predominantly categorized as stage 2 . These findings agree with the results of other studies $[19,21]$.

Almost half of the study participants reported tooth extraction, a known preceding event, prior to the development of MRONJ [19,21]. The pooled analysis of three phase III studies in 5,723 patients with metastatic bone disease receiving BTAs, reported that $62 \%$ of the patients with MRONJ had undergone tooth extraction, with high rates of simultaneously having oral infection and jaw pain [21]. In our study, periodontal disease was the second most frequently reported risk factor, after tooth extraction and tooth pain was reported in almost one out of three patients. This comes in accordance with previous evidence that shows that periodontal infection and pain are significantly associated with jawbone necrosis, prior to the dental extraction, and subsequent MRONJ development $[22,23]$.

In our study CBCT was superior to OPG in interpreting the extent of osteolysis, sclerosis, periosteal reaction and sequestration. Furthermore, CBCT was superior to OPG in disclosing all four predefined imaging characteristics at the early stages 1 and 2 , as well as all characteristics except for periosteal reaction, in stage 3 . CT scans were also reported useful to define the extent of osteolytic changes and could accurately describe the stage of MRONJ in other studies $[6,7,10]$. Furthermore, bone sclerosis, lytic changes, periosteal reaction and sequestration, in both OPG and $\mathrm{CBCT}$, were observed more often as the MRONJ stage advanced by other investigators $[8,9,11]$. The above studies have the following important weaknesses: They were a. retrospective type of study, b. MRONJ staging was set only from clinical evaluation, c. small numbers of patients were included and $\mathrm{d}$. they included mixed populations, osteoporosis and cancer patients [6-11]. Our study was prospective, included homogeneous patient population, MRONJ staging was set by both clinical and radiographic findings and the imaging characteristics were clustered by examination type and by stage.

\section{Conclusion}

The results of our study indicated that CBCT is superior and more stage-sensitive to OPG in interpreting MRONJ. CBCT is particularly useful for early diagnosis and staging MRONJ to achieve effective treatment. CBCT may assist to update the definition of MRONJ to include the non-exposed type of MRONJ, as well as to identify early, prior to dental extraction jawbone necrosis $[20,22,23]$.

\section{References}

1. Ruggiero SL, Dodson TB, Fantasia J, Goodday R, Aghaloo T, Mehrotra B, et al. American Association of Oral and Maxillofacial Surgeons position paper on medication-related osteonecrosis of the jaw--2014 update. J Oral Maxillofac Surg. 2014;72(10):1938-56.

2. Khan AA, Morrison A, Kendler DL, Rizzoli R, Hanley DA, Felsenberg D, et al. Case-based review of Osteonecrosis of the Jaw (ONJ) and Application of the International Recommendations for Management From the International Task Force on ONJ. J Clin Densitom. 2017;20(1):8-24.

3. Otto S, Pautke C, Van den Wyngaert T, Niepel D, Schiødt M. Medicationrelated osteonecrosis of the jaw: Prevention, diagnosis and management in patients with cancer and bone metastases. Cancer Treat Rev. 2018;69:17787.

4. Yoneda T, Hagino H, Sugimoto T, Ohta H, Takahashi S, Soen S. Antiresorptive agent-related osteonecrosis of the jaw: Japanese Allied Committee on Osteonecrosis of the Jaw. Position Paper 2017 of the Japanese Allied Committee on Osteonecrosis of the Jaw. J Bone Miner Metab. 2017;35(1):6-19.

5. Yarom N, Shapiro CL, Peterson DE, Van Poznak CH, Bohlke K, Ruggiero $\mathrm{SL}$, et al. Medication-related osteonecrosis of the Jaw: MASCC/ISOO/ ASCO Clinical Practice Guideline. J Clin Oncol. 2019;37(25):2270-90.

6. Chiandussi S, Biasotto M, Dore F, Cavalli F, Cova MA, Lenarda RD, et al. Clinical and diagnostic imaging of bisphosphonate-associated osteonecrosis of the jaws. Dentomaxillofac Radiol. 2006;35(4):236-43.

7. Bedogni A, Fedele S, Bedogni G, Scoletta M, Favia G, Colella G, et al. Staging of osteonecrosis of the jaw requires computed tomography for accurate definition of the extent of bony disease. Br J Oral Maxillofac Surg. 2014;52(7):603-8.

8. Bagan JV, Cibrian RM, Lopez J, Leopoldo-Rodado M, Carbonell E, Bagán $\mathrm{L}$, et al. Sclerosis in bisphosphonate-related osteonecrosis of the jaws and its correlation with the clinical stages: study of 43 cases. Br J Oral Maxillofac Surg. 2015;53(3):257-62.

9. Cardoso CL, Barros CA, Curra C, Paes da Silva Ramos Fernandes LM, de Oliveira Braga Franzolin S, Ferreira Júnior JS, et al. Radiographic findings in patients with medication-related osteonecrosis of the Jaw. Int J Dent. 2017;2017:3190301.

10. Baba A, Goto TK, Ojiri H, Takagiwa M, Hiraga C, Okamura M, et al. CT imaging features of antiresorptive agent-related osteonecrosis of the jaw/ medication-related osteonecrosis of the jaw. Dentomaxillofacial Radiol. 2018;47(4):20170323.

11. Walton K, Grogan TR, Eshaghzadeh E, Hadaya D, Elashoff DA, Aghaloo TL, et al. Medication-related osteonecrosis of the jaw in osteoporotic vs. oncologic patients-quantifying radiographic appearance and relationship to clinical findings. Dentomaxillofacial Radiology. 2019;48(1):20180128.

12. Takaishi Y, Ikeo T, Nakajima M, Miki T, Fujita T. A pilot case-control study on the alveolar bone density measurement in risk assessment for bisphosphonate-related osteonecrosis of the jaw. Osteoporos Int. 2010;21(5):815-25.

13. Fleisher KE, Welch G, Kottal S, Craig RG, Saxena D, Glickman RS, et al. Predicting risk for bisphosphonate-related osteonecrosis of the jaws: CTX versus radiographic markers. Oral Surg Oral Med Oral Pathol Oral Radiol Endod. 2010;110(4):509-16.

14. Walter C, Laux C, Sagheb K. Radiologic bone loss in patients with bisphosphonate-associated osteonecrosis of the jaws: A case-control study. Clin Oral Investig. 2014;18(2):385-90.

15. Soundia A, Hadaya D, Mallya SM, Aghaloo TL, Tetradis S. Radiographic predictors of bone exposure in patients with stage 0 medication-related osteonecrosis of the jaws. Oral Surg Oral Med Oral Pathol Oral Radiol. 2018;126(6):537-44.

16. Leite AF, Ogata Fdos S, de Melo NS, de Souza Figueiredo PT. Imaging 
findings of bisphosphonate-related osteonecrosis of the jaws: A critical review of the quantitative studies. Int J Dent. 2014;11:784348.

17. Dutra KL, Haas LF, Zimmermann GS, Zimmermann GS, Melo G, Minamisako MC, et al. Prevalence of radiographic findings on jaws exposed to antiresorptive therapy: A meta-analysis. Dentomaxillofac Radiol. 2019;48(3):20180112.

18. StataCorp. Stata Statistical Software Release 15 College Station TX StataCorp LLC. 2017.

19. Schiodt M, Vadhan-Raj S, Chambers MS, Nicolatou-Galitis O, Politis C, Coropciuc R, et al. A multicenter case registry study on medication-related osteonecrosis of the jaw in patients with advanced cancer. Support Care Cancer. 2018;26(6):1905-15.

20. Schiodt M, Otto S, Fedele S, Bedogni A, Nicolatou-Galitis O, Guggenberger $\mathrm{R}$, et al. Workshop of European Task Force on Medication Related Osteonecrosis of the Jaw - Current Challenges. Oral Dis. 2019;25(7):181521.
21. Saad F, Brown JE, Van Poznak C, Ibrahim T, Stemmer SM, Stopeck AT, et al. Incidence, risk factors, and outcomes of osteonecrosis of the jaw: Integrated analysis from three blinded active-controlled phase III trials in cancer patients with bone metastases. Ann Oncol. 2012;23(5):1341-7.

22. Nicolatou-Galitis O, Papadopoulou E, Vardas E, Kouri M, Galiti D, Galitis $\mathrm{E}$, et al. Alveolar bone histological necrosis observed prior to extractions in patients, who received bone-targeting agents. Oral Dis. 2020;26(5):955-66.

23. Ristow O, Ruckschlob T, Moratin J, Müller M, Kühle R, Dominik H, et al. Wound closure and alveoplasty after preventive tooth extractions in patients with antiresorptive intake-A randomized pilot trial. Oral Dis. 2021;27(3):532-46. 